Karlsruhe Reports in Informatics 2010,2 Edited by Karlsruhe Institute of Technology, Faculty of Informatics ISSN 2190-4782

\title{
Path Schematization for Route Sketches
}

Daniel Delling, Andreas Gemsa,

Martin Nöllenburg, Thomas Pajor

2010

KIT - University of the State of Baden-Wuerttemberg and National Research Center of the Helmholtz Association 


\section{Fakultät für Informatik

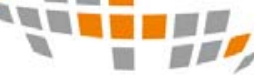 \\ 밈}

\section{Please note:}

This Report has been published on the Internet under the following Creative Commons License:

http://creativecommons.org/licenses/by-nc-nd/3.0/de. 


\title{
Path Schematization for Route Sketches
}

\author{
Daniel Delling ${ }^{1}$, Andreas Gemsa $^{2}$, Martin Nöllenburg ${ }^{2,3 \star}$, and Thomas Pajor ${ }^{2}$ \\ 1 Microsoft Research Silicon Valley, 1065 La Avenida, Mountain View, CA 94043. \\ dadellin@microsoft.com \\ 2 Karlsruhe Institute of Technology, P.O. Box 6980, 76128 Karlsruhe, Germany. \\ \{gemsa, noellenburg, pajor\}@kit.edu \\ 3 Department of Computer Science, University of California, Irvine, CA 92697-3435.
}

\begin{abstract}
Motivated from drawing route sketches, we consider the following path schematization problem. We are given a simple embedded polygonal path $P=\left(v_{1}, \ldots, v_{n}\right)$ and a set $\mathcal{C}$ of admissible edge orientations including the coordinate axes. The problem is to redraw $P$ schematically such that all edges are drawn as line segments that are parallel to one of the specified orientations. We also require that the path preserves the orthogonal order and that it remains intersection-free. Finally, we want the drawing to maximize the number of edges having their preferred edge direction and to minimize the path length.

In this paper we first present an efficient two-step approach for schematizing monotone paths. It consists of an $O\left(n^{2}\right)$-time algorithm to assign edge directions optimally and a subsequent linear program to minimize the path length. In order to schematize non-monotone paths we propose a heuristic that first splits the input into $k$ monotone subpaths and then combines the optimal embeddings of the monotone subpaths into a single, intersection-free embedding of the initial path in $O\left(k^{2}+n\right)$ time.
\end{abstract}

\section{Introduction}

Simplification and schematization of map objects are well-known operators in cartographic generalization, i. e., the process to adapt map content to its scale and use. Simplification usually reduces unnecessary complexity, e. g., by removing extraneous vertices of a polygonal line while still maintaining its overall appearance. Schematization, however, may abstract more drastically from geographic reality as long as the intended map use allows for it. Public transport maps are good examples of schematization, where edge orientations are limited to a small number of slopes and edge lengths are no longer drawn to scale [8]. In spite of all distortions, such maps usually work well.

In this paper we consider a path schematization problem that is motivated from visualizing routes in road networks. Routes typically begin and end in residential or commercial areas, where roads are mostly used only for short distances of a few meters up to a few hundred meters. As soon as the route leaves the city limits, however, country roads and highways tend to be used for distances

\footnotetext{
* Supported by grant NO 899/1-1 of the German Research Foundation (DFG).
} 


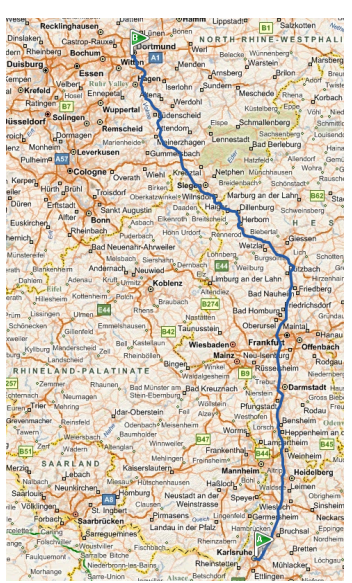

(a) Route in Bing Maps.

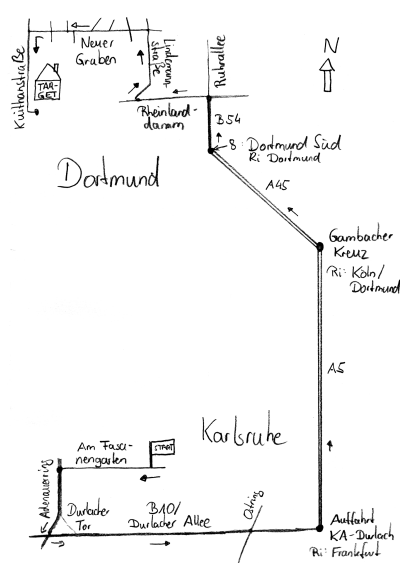

(b) Hand-drawn sketch.

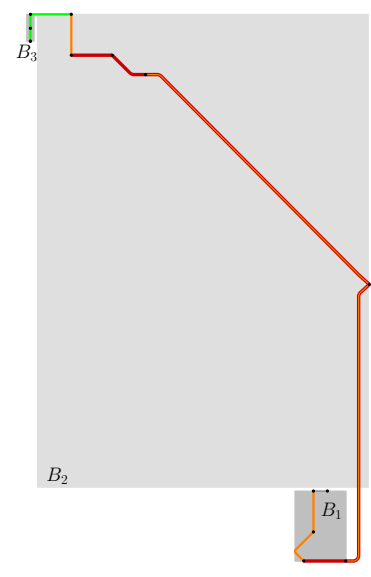

(c) Output of our algorithm.

Fig. 1: Comparison of different methods for drawing a route from Karlsruhe to Dortmund in Germany. In Figure 1c, different colors indicate different road categories. $B_{i}$ indicate the bounding boxes of the monotone subpaths.

ranging from a few up to hundreds of kilometers. Moreover, optimal routes tend to follow a general driving direction and deviations from this direction are rare.

Commercial route planners typically present driving directions for such routes as a graphical overview of the route highlighted in a traditional road map (see Fig. 1a) in combination with a textual step-by-step description. The overview map is good for giving a general idea of the route, but due to its small scale it often does not succeed in showing details of the route, in particular for short roads in the vicinity of start and destination and off the main highways. Textual descriptions are accurate when used at the right moment but there is a high risk of loss of context. On the other hand, a manually drawn route sketch often shows the whole route in a single picture, where each part of the route has its own appropriate scale: important turning points along the route and short residential roads are enlarged while long stretches of highways and country roads are shortened. Edges are often aligned with a small set of orientations rather than being geographically accurate [10]. Figure $1 \mathrm{~b}$ gives an example. In spite of the cartographic error, such route sketches are often easier to read than textual descriptions and traditional road maps - at least if the user's mental or cognitive map, i. e., a rough idea of the geographic reality, is preserved [6,9].

We formalize the application problem of drawing route sketches as a geometric path schematization problem. Given a plane embedding of a path $P$, the goal is to find a short schematic embedding of $P$ that is as similar to the input embedding as possible but uses only a restricted set $\mathcal{C}$ of edge orientations. We call such an embedding $\mathcal{C}$-oriented. For our application of route sketches, the 
path $P$ is given by $n$ important points along the route. These important points can be turns, important junctions, highway ramps, etc.

Related Work. Similar path schematization problems have been studied before. Neyer [7] proposed an algorithm to solve the $\mathcal{C}$-oriented line simplification problem, where a $\mathcal{C}$-oriented simplification $Q$ of a polygonal path $P$ is to be computed that uses a minimum number of edges. Furthermore, $Q$ must have Fréchet distance at most $\varepsilon$ from $P$. For a constant-size set $\mathcal{C}$ the algorithm has a running time of $O\left(k n^{2} \log n\right)$, where $n$ is the number of vertices of $P$ and $k$ is the number of vertices of $Q$.

Merrick and Gudmundsson [5] studied a slightly relaxed version of the same problem and gave an $O\left(n^{2}|\mathcal{C}|^{3}\right)$-time algorithm to compute a $\mathcal{C}$-oriented simplification of $P$ that is within Hausdorff distance at most $\varepsilon$ of $P$.

Agrawala and Stolte [1] designed a system called LineDrive that uses heuristic methods based on simulated annealing in order to render route maps similar to hand-drawn sketches. While their system allows distortion of edge lengths and angles, the resulting paths are neither $\mathcal{C}$-oriented nor can hard quality guarantees be given. They did, however, implement and evaluate the system in a study that showed that users generally preferred LineDrive route maps over traditional route maps.

Brandes and Pampel [2] studied the path schematization problem in the presence of orthogonal order constraints [6] in order to preserve the mental map. They showed that deciding whether a rectilinear schematization of a path $P$ exists that preserves the orthogonal order of $P$ is NP-hard. They also showed that schematizing a path using arbitrarily oriented unit-length edges is NP-hard.

Our Contribution. Due to the NP-hardness of rectilinear path schematization [2], we cannot hope for an algorithm that solves the general $\mathcal{C}$-oriented path schematization problem efficiently. Rather, we present an efficient algorithm to solve the corresponding monotone path schematization problem, in which the input is restricted to $x$ - or $y$-monotone paths (Section 3). The algorithm consists of two steps: First, we compute in quadratic time a $\mathcal{C}$-oriented schematization of the input path that preserves the orthogonal order of the input and has minimum schematization cost (to be defined). Next, we use a simple linear program to minimize the total path length such that the schematization cost remains minimum.

In order to use this algorithm to generate route sketches for non-monotone input paths, we present a three-step heuristic approach (Section 4): We first split the path in linear time into a minimum number $k$ of monotone subpaths, then we use the previous algorithm to optimally schematize each subpath, and finally we combine the $k$ schematized subpaths into a single intersection-free route sketch for the non-monotone input path in $O\left(k^{2}+n\right)$ time. Note that routes in practice tend to follow a general direction given by the straight line connecting start and destination. Thus if a path is not monotone itself, then it usually consists of a very small number of monotone subpaths (see the example in Fig. 1c, which decomposes into three monotone subpaths). 


\section{Preliminaries}

Let $P=\left(v_{1}, \ldots, v_{n}\right)$ be a path with edges $v_{i} v_{i+1}$ for $1 \leq i \leq n-1$. For a vertex $v$ and an edge $e$ of $P$ we say $v \in P$ and $e \in P$. A plane embedding $\pi: P \rightarrow \mathbb{R}^{2}$ maps each vertex $v_{i} \in P$ to a point $\pi\left(v_{i}\right)=\left(x_{\pi}\left(v_{i}\right), y_{\pi}\left(v_{i}\right)\right)$ and each edge $u v \in P$ to the line segment $\pi(u v)=\overline{\pi(u) \pi(v)}$ such that $\pi$ is a simple polygonal path with vertex set $\left\{\pi\left(v_{1}\right), \ldots, \pi\left(v_{n}\right)\right\}$. We denote the length of an edge $e$ in $\pi$ as $|\pi(e)|$. An embedded path is a pair $(P, \pi)$ of a path $P$ and a plane embedding $\pi$ of $P$.

Let $\mathcal{C}=\left\{\gamma_{1}, \ldots, \gamma_{k}\right\}$ be a set of angles w.r.t. the $x$-axis that represents the admissible edge orientations. We require that $\left\{0^{\circ}, 90^{\circ}, 180^{\circ}, 270^{\circ}\right\} \subseteq \mathcal{C}$. Reasonable sets of edge directions for route sketches are, e. g., multiples of 30 or 45 degrees. Recall that a plane embedding $\pi$ of a path is called $\mathcal{C}$-oriented if the direction of each edge in $\pi$ corresponds to an angle in $\mathcal{C}$. For an embedding $\pi$ of $P$ and an edge $e \in P$ we denote by $\alpha_{\pi}(e)$ the angle of $\pi(e)$ w.r.t. the $x$-axis. For the input embedding $\pi$, we similarly denote by $\omega_{\mathcal{C}}(e)$ the preferred angle $\gamma \in \mathcal{C}$, i. e., the angle in $\mathcal{C}$ that is closest to $\alpha_{\pi}(e)$. For a $\mathcal{C}$-oriented embedding $\rho$ of $P$ and an edge $e \in P$ there is a direction cost $c_{\rho}(e)$ that captures by how much the angle $\alpha_{\rho}(e)$ deviates from $\omega_{\mathcal{C}}(e)$. The schematization cost $c(\rho)$ is then defined as $c(\rho)=\sum_{e \in P} c_{\rho}(e)$.

Following Misue et al. [6], we say that an embedding $\rho$ of a path $P$ preserves the orthogonal order of another embedding $\pi$ of $P$ if for any two vertices $v_{i}$ and $v_{j} \in P$ we have $x_{\pi}\left(v_{i}\right) \leq x_{\pi}\left(v_{j}\right)$ if and only if $x_{\rho}\left(v_{i}\right) \leq x_{\rho}\left(v_{j}\right)$ and $y_{\pi}\left(v_{i}\right) \leq y_{\pi}\left(v_{j}\right)$ if and only if $y_{\rho}\left(v_{i}\right) \leq y_{\rho}\left(v_{j}\right)$. In other words, any two vertices keep their abovebelow and left-right relationship.

\section{Monotone Path Schematization}

In this section, we solve the monotone $\mathcal{C}$-oriented path schematization problem, which is formally defined as follows.

Problem 1. Given an embedded $x$ - or $y$-monotone path $(P, \pi)$, a set $\mathcal{C}$ of edge orientations and a minimum length $\ell_{\min }(e)$ for each edge $e \in P$, find a plane $\mathcal{C}$-oriented embedding $\rho$ of $P$ that

(i) preserves the orthogonal order of the input embedding $\pi$,

(ii) minimizes the schematization cost $c(\rho)$,

(iii) respects the individual minimum edge lengths $|\rho(e)| \geq \ell_{\min }(e)$, and

(iv) minimizes the total path length $\sum_{e \in P}|\rho(e)|$.

Note that schematization cost and total path length are two potentially conflicting optimization criteria. Primarily, we want to find an embedding that minimizes the schematization cost (see Section 3.1). In a second step, we minimize the total path length of that embedding without changing the previously assigned edge directions (see Section 3.2). The rationale for preserving the orthogonal order of the input is to maintain the user's mental map $[2,4,6]$. 


\subsection{Minimizing the Schematization Cost}

The goal in the first step of our algorithm is to find an embedding with minimum schematization cost. Here we assume that the input path $(P, \pi)$ is $x$-monotone; $y$-monotone paths are schematized analogously. We assign the preferred angle $\omega_{\mathcal{C}}(e)=\gamma$ to each edge $e \in P$, where $\gamma \in \mathcal{C}$ is the angle closest to $\alpha_{\pi}(e)$. This takes constant time per edge. It could, however, result in the following conflict. Consider two subsequent edges $e_{1}, e_{2}$ with $\left\{\omega_{\mathcal{C}}\left(e_{1}\right),\left(\omega_{\mathcal{C}}\left(e_{2}\right)\right\}=\left\{90^{\circ}, 270^{\circ}\right\}\right.$. Assigning such preferred angles would result in an overlap of $e_{1}$ and $e_{2}$. In this case, we either set $\omega_{\mathcal{C}}\left(e_{1}\right)$ or $\omega_{\mathcal{C}}\left(e_{2}\right)$ to its next best value, depending on which edge is closer to it. This neither changes the solution nor creates new conflicts since in a plane embedding not both edges can have their preferred direction.

The output embedding $\rho$ must be $x$-monotone, too, as it preserves the orthogonal order of $\pi$. So we can assume that $P=\left(v_{1}, \ldots, v_{n}\right)$ is ordered from left to right in both embeddings. Let $\rho^{\prime}$ be any orthogonal-order preserving embedding of $P$. We start with the observation that in $\rho^{\prime}$ every edge $e=v_{i} v_{i+1}$ with $\omega_{\mathcal{C}}(e) \neq 0^{\circ}$ and $y_{\rho^{\prime}}\left(v_{i}\right) \neq y_{\rho^{\prime}}\left(v_{i+1}\right)$ can be embedded with its preferred direction $\alpha_{\rho^{\prime}}(e)=\omega_{\mathcal{C}}(e)$. This is achieved by horizontally shifting the whole embedding $\rho^{\prime}$ right of $x_{\rho^{\prime}}\left(v_{i+1}\right)$ (including $\left.v_{i+1}\right)$ to the left or to the right until the slope of $e$ satisfies $\alpha_{\rho^{\prime}}(e)=\omega_{\mathcal{C}}(e)$. Due to the $x$-monotonicity of $P$ no other edges are affected by this shift.

We now group all edges $e=u v$ of $P$ into four categories according to their preferred angle $\omega_{\mathcal{C}}(e)$ :

1. if $\omega_{\mathcal{C}}(e)=0^{\circ}$ and $y_{\pi}(u) \neq y_{\pi}(v)$ then $e$ is called horizontal edge (or h-edge);

2. if $y_{\pi}(u)=y_{\pi}(v)$ then $e$ is called strictly horizontal edge (or sh-edge);

3. if $\omega_{\mathcal{C}}(e) \neq 0^{\circ}$ and $x_{\pi}(u) \neq x_{\pi}(v)$ then $e$ is called vertical edge (or $v$-edge);

4. if $x_{\pi}(u)=x_{\pi}(v)$ then $e$ is called strictly vertical edge (or sv-edge).

Using these categories, we define the direction cost as follows. All edges $e$ with $\alpha_{\rho}(e)=\omega_{\mathcal{C}}(e)$ are drawn according to their preferred angle and we assign the cost $c_{\rho}(e)=0$. For all edges $e$ with $\alpha_{\rho}(e) \neq \omega_{\mathcal{C}}(e)$ we assign the cost $c_{\rho}(e)=1$. An exception are the sh- and sv-edges, which must be assigned their preferred angle due to the orthogonal ordering constraints. Consequently, we set $c_{\rho}(e)=$ $\infty$ for any sh- or sv-edge $e$ with $\alpha_{\rho}(e) \neq \omega_{\mathcal{C}}(e)$. Using the above horizontal shifting argument, the cost $c_{\rho}(e)$ of any edge $e$ depends only on the vertical distance between its endpoints. Hence, the schematization cost of an $x$-monotone embedding $\rho$ is already fully determined by assigning $y$-coordinates $y_{\rho}(v)$ to all vertices $v$ of $P$. Note that our approach can be easily adapted to alternative cost definitions.

In order to determine an embedding with minimum schematization cost we define $m \leq n-1$ closed and vertically bounded horizontal strips $s_{1}, \ldots, s_{m}$ induced by the set $\left\{y=y_{\pi}\left(v_{i}\right) \mid 1 \leq i \leq n\right\}$ of horizontal lines through the vertices of $(P, \pi)$. Let these strips be ordered from top to bottom as shown in Fig. 2a. Furthermore we define a dummy strip $s_{0}$ above $s_{1}$ that is unbounded on its upper side. We say that an edge $e=u v$ crosses a strip $s_{i}$ and conversely that $s_{i}$ affects $e$ if $\pi(u)$ and $\pi(v)$ lie on opposite sides of $s_{i}$. In fact, to determine 


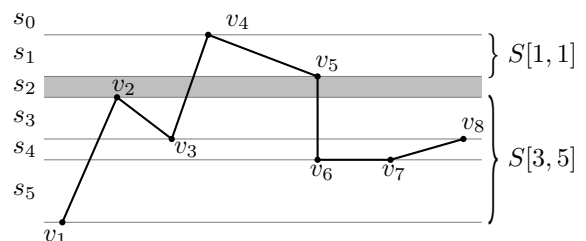

(a) Horizontal strips for $(P, \pi)$.

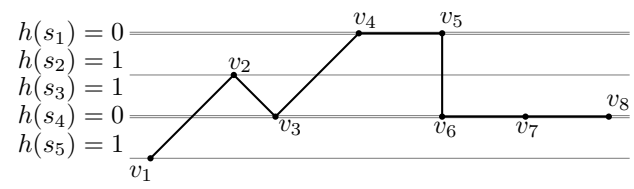

(b) Strip height assignment and induced output path $(P, \rho)$.

Fig. 2: Example of (a) an $x$-monotone embedded input path $(P, \pi)$ and (b) a $\mathcal{C}$-oriented orthogonal-order preserving output path $(P, \rho)$, where $\mathcal{C}$ contains all multiples of $45^{\circ}$.

the cost of an embedding $\rho$ it is enough to know for each strip whether it has a positive height or not. Our algorithm will assign a symbolic height $h\left(s_{i}\right) \in\{0,1\}$ to each strip $s_{i}$ such that the schematization cost is minimum. Note that sh-edges do not cross any strip but rather coincide with some strip boundary. Hence all sh-edges are automatically drawn horizontally and have no direction costs. We can therefore assume that there are no sh-edges in $(P, \pi)$.

Let $S[i, j]=\bigcup_{k=i}^{j} s_{k}$ be the union of the strips $s_{i}, \ldots, s_{j}$ and let $\mathcal{I}(i, j)$ be the subinstance of the path schematization problem containing all edges that lie completely within $S[i, j]$. Note that $\mathcal{I}(1, m)$ corresponds to the original instance $(P, \pi)$, whereas in general $\mathcal{I}(i, j)$ is no longer a connected path but a collection of edges. The following lemma is a key to our algorithm.

Lemma 1. Let $\mathcal{I}(i, j)$ be a subinstance of the path schematization problem and let $s_{k} \subseteq S[i, j]$ be a strip for some $i \leq k \leq j$. If we assign $h\left(s_{k}\right)=1$ then $\mathcal{I}(i, j)$ decomposes into the two independent subinstances $\mathcal{I}(i, k-1)$ and $\mathcal{I}(k+1, j)$. The direction costs of all edges affected by $s_{k}$ are determined by setting $h\left(s_{k}\right)=1$.

Proof. We first show that the cost of any edge $e=u v$ that crosses $s_{k}$ is determined by setting $h\left(s_{k}\right)=1$. Since $u$ and $v$ lie on opposite sides of $s_{k}$ we know that $y_{\rho}(u) \neq y_{\rho}(v)$. So if $e$ is a v- or sv-edge it can be drawn with its preferred angle and $c_{\rho}(e)=0$ regardless of the height of any other strip crossed by $e$. Conversely, if $e$ is an h-edge it is impossible to draw $e$ horizontally regardless of the height of any other strip crossed by $e$ and $c_{\rho}(e)=1$. Recall that sh-edges do not cross any strips. Assume that $k=2$ in Fig. $2 \mathrm{a}$ and we set $h\left(s_{2}\right)=1$; then edges $v_{3} v_{4}$ and $v_{5} v_{6}$ cross strip $s_{2}$ and none of them can be drawn horizontally.

The remaining edges of $\mathcal{I}(i, j)$ do not cross $s_{k}$ and are either completely contained in $S[i, k-1]$ or in $S[k+1, j]$. Since the costs of all edges affected by $s_{k}$ are independent of the heights of the remaining strips in $S[i, j] \backslash\left\{s_{k}\right\}$, we can solve the two subinstances $\mathcal{I}(i, k-1)$ and $\mathcal{I}(k+1, j)$ independently, see Fig. 2a.

Our Algorithm. We can now describe our algorithm for assigning symbolic heights to all strips $s_{1}, \ldots, s_{m}$ such that the induced embedding $\rho$ has minimum schematization cost. The main idea is to recursively compute an optimal 
solution for each instance $\mathcal{I}(1, i)$ by finding the best $k \leq i$ such that $h\left(s_{k}\right)=1$ and $h\left(s_{j}\right)=0$ for $j=k+1, \ldots, i$. By using dynamic programming we can compute an optimal solution for $\mathcal{I}(1, m)=(P, \pi)$ in $O\left(n^{2}\right)$ time.

Let $C(k, i)$ for $1 \leq k \leq i$ denote the schematization cost of all edges in the instance $\mathcal{I}(1, i)$ that either cross $s_{k}$ or have both endpoints in $S[k+1, i]$ if we set $h\left(s_{k}\right)=1$ and $h\left(s_{j}\right)=0$ for $j=k+1, \ldots, i$. Let $C(0, i)$ denote the schematization cost of all edges in the instance $\mathcal{I}(1, i)$ if $h\left(s_{j}\right)=0$ for all $j=1, \ldots, i$. We use an array $T$ of size $m+2$ to store the minimum schematization cost $T[i]$ of the instance $\mathcal{I}(1, i)$. Then $T[i]$ is recursively defined as follows

$$
T[i]= \begin{cases}\min _{0 \leq k \leq i}(T[k-1]+C(k, i)) & \text { if } 1 \leq i \leq m \\ 0 & \text { if } i=0 \text { or } i=-1 .\end{cases}
$$

Together with $T[i]$ we store the index $k$ that achieves the minimum value in the recursive definition of $T[i]$ as $k[i]=k$. This allows us to compute the actual strip heights using backtracking. Note that $T[m]<\infty$ since, e.g., the solution that assigns height 1 to every strip induces cost 0 for all sv-edges. Obviously, we need $O(m)$ time to compute each entry in $T$ assuming that the schematization cost $C(k, i)$ is available in $O(1)$ time. This yields a total running time of $O\left(\mathrm{~m}^{2}\right)$.

The next step is to precompute the schematization cost $C(k, i)$ for any $0 \leq k \leq i \leq m$. This cost is composed of two parts. The first part is the schematization cost of all edges that are affected by $s_{k}$. As observed in Lemma 1, all v- and sv-edges crossing $s_{k}$ have no cost. On the other hand, every h-edge that crosses $s_{k}$ has cost 1 . So we need to count all h-edges in $\mathcal{I}(1, i)$ that cross $s_{k}$. The second part is the cost of all edges that are completely contained in $S[k+1, i]$. Since $h\left(s_{k+1}\right)=\ldots=h\left(s_{i}\right)=0$ we observe that any h-edge in $S[k+1, i]$ is drawn horizontally at no cost. In contrast, no v- or sv-edge $e$ in $S[k+1, i]$ attains its preferred angle $\omega_{\mathcal{C}}(e) \neq 0^{\circ}$. Hence every v-edge in $S[k+1, i]$ has cost 1 and every sv-edge has cost $\infty$. So we need to check whether there is an sv-edge contained in $S[k+1, i]$ and if this is not the case count all v-edges contained in $S[k+1, i]$.

In order to efficiently compute the values $C(k, i)$ we assign to each strip $s_{i}$ three sets of edges. Let $H(i)$ (resp. $V(i)$ or $S V(i)$ ) be the set of all h-edges (resp. v-edges or sv-edges) whose lower endpoint lies on the lower boundary of $s_{i}$. We can compute $H(i), V(i)$, and $S V(i)$ in $O(n)$ time for all strips $s_{i}$. Then for $k \leq i$ the number of h-edges in $H(i)$ that cross $s_{k}$ is denoted by $\sigma_{H}(k, i)$ and the number of v-edges in $V(i)$ that do not cross $s_{k}$ is denoted by $\sigma_{V}(k, i)$. Finally, let $\sigma_{S V}(k, i)$ be the number of sv-edges in $S V(i)$ that do not cross $s_{k}$.

This allows us to compute the values $C(k, i), 0 \leq k \leq i \leq m$, recursively as follows

$$
C(k, i)= \begin{cases}\infty & \text { if } \sigma_{S V}(k, i) \geq 1 \\ C(k, i-1)+\sigma_{H}(k, i)+\sigma_{V}(k, i) & \text { if } k \leq i-1 \\ \sigma_{H}(k, k) & \text { if } k=i\end{cases}
$$

Since each edge appears in exactly one of the sets $H(i), V(i)$, or $S V(i)$ for some $i$ it is counted towards at most $m$ values $\sigma_{H}(\cdot, i), \sigma_{V}(\cdot, i)$, or $\sigma_{S V}(\cdot, i)$, respectively. 
Thus for computing all these values we need $O(n m)$ time. The values $C(k, i)$ can be precomputed in $O\left(\mathrm{~m}^{2}\right)$ time and require a table of size $O\left(\mathrm{~m}^{2}\right)$. This can be reduced, however, to $O(m)$ space as follows. We compute and store the values $T[i]$ in the order $i=1, \ldots, m$. For computing the entry $T[i]$ we use only the values $C(\cdot, i)$. To compute the next entry $T[i+1]$ we first compute the values $C(\cdot, i+1)$ from $C(\cdot, i)$ and then discard all $C(\cdot, i)$. This reduces the required space to $O(m)$. Since $m \leq n$ we obtain

Theorem 1. Our algorithm to compute the array $T$ of path schematization costs requires $O\left(n^{2}\right)$ time and $O(n)$ space.

It remains to determine the strip height assignments corresponding to the schematization cost in $T[m]$ and show the optimality of that solution. We initialize all heights $h\left(s_{i}\right)=0$ for $i=1, \ldots, m$. Recall that $k[i]$ equals the index $k$ that minimized the value $T[i]$ in (1). To find all strips with height 1 we initially set $j=m$. If $k[j]=0$ we stop; otherwise we assign $h\left(s_{k[j]}\right)=1$, update $j=k[j]-1$, and continue with the next index $k[j]$ until we hit $k[j]=0$ for some $j$ encountered in this process. Let $\rho$ be the $\mathcal{C}$-oriented embedding of $P$ induced by this strip height assignment, see Fig. 2 b. We now show the optimality of $\rho$ in terms of the schematization cost.

Theorem 2. Given an $x$-monotone embedded path $(P, \pi)$ and a set $\mathcal{C}$ of edge orientations, our algorithm computes a plane $\mathcal{C}$-oriented embedding $\rho$ of $P$ that preserves the orthogonal order of $\pi$ and has minimum schematization cost $c(\rho)$.

Proof. Since the path is $x$-monotone and by construction there are no two adjacent edges with preferred angles $90^{\circ}$ and $270^{\circ}$ the embedding $\rho$ is plane. Furthermore, the $x$-coordinates of the vertices are chosen such that $\rho$ is $\mathcal{C}$-oriented, see Fig. 2b. The embedding $\rho$ also preserves the orthogonal order of $\pi$ since $\rho$ does not alter the $x$ - and $y$-ordering of the vertices of $P$.

We show that $\rho$ has minimum schematization cost by structural induction. For an instance with a single strip $s$ there are only two possible solutions of which our algorithm chooses the better one. The induction hypothesis is that our algorithm finds an optimal solution for any instance with at most $m$ strips. So let's consider an instance with $m+1$ strips and let $\rho^{\prime}$ be any optimal plane $\mathcal{C}$ oriented and orthogonal-order preserving solution for this instance. If all strips $s$ in $\rho^{\prime}$ have height $h(s)=0$ then by (1) it holds that $c(\rho)=T[m+1] \leq$ $C(0, m+1)=c\left(\rho^{\prime}\right)$. Otherwise, let $k$ be the largest index for which $h\left(s_{k}\right)=1$ in $\rho^{\prime}$. When computing $T[m+1]$ our algorithm also considers the case where $s_{k}$ is the bottommost strip of height 1 , which has a cost of $T[k-1]+C(k, m+1)$. If $h\left(s_{k}\right)=1$ we can split the instance into two independent subinstances to both sides of $s_{k}$ by Lemma 1 . The schematization cost $C(k, m+1)$ contains the cost for all edges that cross $s_{k}$ and this cost is obviously the same as in $\rho^{\prime}$ since $h\left(s_{k}\right)=1$ in both embeddings. Furthermore, $C(k, m+1)$ contains the cost of all edges in the subinstance below $s_{k}$, for which we have by definition $h\left(s_{k+1}\right)=\ldots=h\left(s_{m+1}\right)=0$. Since $k$ is the largest index with $h\left(s_{k}\right)=1$ in $\rho^{\prime}$ this is also exactly the same cost that this subinstance has in $\rho^{\prime}$. Finally, 
the independent subinstance above $s_{k}$ has at most $m$ strips and hence $T[k-1]$ is the minimum cost for this subinstance by induction. It follows that $c(\rho)=$ $T[m+1] \leq T[k-1]+C(k, m+1) \leq c\left(\rho^{\prime}\right)$. This concludes the proof.

\subsection{Minimizing the Path Length}

In the first step of our algorithm we obtained a $\mathcal{C}$-oriented and orthogonal-order preserving embedding $\rho$ with minimum schematization cost for an embedded input path $(P, \pi)$. The strip heights assigned in that step are either 0 or 1 , but this does not yet take into account the actual edge lengths induced by $\rho$. So in the second step, we adjust $\rho$ such that the total path length is minimized and $|\rho(e)| \geq \ell_{\min }(e)$ for all $e \in P$. We make sure, however, that the orthogonal order and all angles $\alpha_{\rho}(e)$-and thus also the schematization cost $c(\rho)$-remain unchanged.

Note that we can immediately assign the minimum length $\ell_{\min }(e)$ to every horizontal edge $e$ in the input $(P, \rho)$ by horizontally shifting the subpaths on both sides of $e$. For any non-horizontal edge $e=u v$ the length $|\rho(e)|$ depends only on the vertical distance $\Delta_{y}(e)=\left|y_{\rho}(u)-y_{\rho}(v)\right|$ of its endpoints and the angle $\alpha_{\rho}(e)$. In fact, $|\rho(e)|=\Delta_{y}(e) / \sin \alpha_{\rho}(e)$. So in order to minimize the path length we need to find $y$-coordinates for all strip boundaries such that $\sum_{e \in P}|\rho(e)|$ is minimized. These $y$-coordinates together with the given angles for all edges $e \in P$ (as computed in the previous step) induce the corresponding $x$-coordinates of all vertices of $P$.

So for each strip $s_{i}(i=0, \ldots, m)$ let $y_{i}$ denote the $y$-coordinate of its lower boundary. For every edge $e \in P$ let $t(e)$ and $b(e)$ denote the index of the top- and bottommost strip, respectively, that is crossed by $e$. Then $\Delta_{y}(e)=y_{t(e)-1}-y_{b(e)}$. We propose the following linear program (LP) to minimize the path length of a given $\mathcal{C}$-oriented embedded path $(P, \rho)$.

$$
\begin{aligned}
& \text { Minimize } \sum_{e \in P, \alpha_{\rho}(e) \neq 0^{\circ}}\left[\frac{1}{\sin \alpha_{\rho}(e)} \cdot\left(y_{t(e)-1}-y_{b(e)}\right)\right] \\
& \text { subject to } \quad y_{t(e)-1}-y_{b(e)} \geq \sin \alpha_{\rho}(e) \cdot \ell_{\min }(e) \quad \forall e \in P, \alpha_{\rho}(e) \neq 0^{\circ} \\
& y_{i-1}-y_{i} \geq 0 \quad \forall s_{i} \text { with } h\left(s_{i}\right)=1 \\
& y_{i-1}-y_{i}=0 \quad \forall s_{i} \text { with } h\left(s_{i}\right)=0
\end{aligned}
$$

We assign to all vertices their corresponding $y$-coordinates from the solution of the LP. In a left-to-right pass over $P$ we compute the correct $x$-coordinates of each vertex $v_{i}$ from the vertical distance to its predecessor vertex $v_{i-1}$ and the angle $\alpha_{\rho}\left(v_{i-1} v_{i}\right)$. This yields a modified embedding $\rho^{\prime}$ that satisfies all our requirements: the path length is minimized; the orthogonal order is preserved due to the $x$-monotonicity of $P$ and the constraints in the LP to maintain the $y$-order; by construction the directions of all edges are the same in $\rho$ and $\rho^{\prime}$; no edge $e$ is shorter than its minimum length $\ell_{\min }(e)$. Hence, $\rho^{\prime}$ solves Problem 1 and together with Theorems 1 and 2 we obtain 
Theorem 3. The monotone $\mathcal{C}$-oriented path schematization problem (Problem 1) for a monotone input path of length $n$ can be solved by an $O\left(n^{2}\right)$-time algorithm to compute an embedding $\rho$ of minimum schematization cost followed by solving a linear program to minimize the path length of $\rho$.

Note that our linear program can be solved efficiently in $O\left(n^{2.5} L\right)$ time using a method of Vaidya [11], where $n$ is the length of the path $P$ and $L$ is the number of input bits.

\section{Extension to General Simple Paths}

In the last section, we showed how to schematize a monotone path. Unfortunately, some routes in road networks are neither $x$ - nor $y$-monotone, however, they can be decomposed into a (very) limited number of $x$ - and $y$ - monotone subpaths. So, we propose the following three-step heuristic to schematize general simple paths: We first split the input path $(P, \pi)$ into a minimum number of $x$ or $y$-monotone subpaths $\left(P_{i}, \pi_{i}\right)$, where $\pi_{i}$ equals $\pi$ restricted to the subpath $P_{i}$. We embed each $\left(P_{i}, \pi_{i}\right)$ separately according to Section 3 . Then, we concatenate the subpaths such that the resulting path $\left(P^{\prime}, \xi\right)$ is a simple $\mathcal{C}$-oriented path. Note that this heuristic does not guarantee to preserve the orthogonal order between node pairs of different subpaths.

Splitting an embedded simple path $P=\left(v_{1}, \ldots, v_{n}\right)$ into the minimal number $k$ of subpaths $P_{i}, 1 \leq i \leq k$ with the property that each $P_{i}$ is an $x$ - or $y$-monotone path can be done in straightforward greedy fashion, starting from $v_{1}$. We traverse $P$ until we find the last vertices $v^{\prime}$ and $v^{\prime \prime}$ which are not violating the $x$ - and $y$-monotonicity, respectively. If $v^{\prime}$ appears later than $v^{\prime \prime}$ on $P$, we set $P_{1}=\left(v_{1}, \ldots, v^{\prime}\right)$, otherwise $P_{1}=\left(v_{1}, \ldots, v^{\prime \prime}\right)$. We continue this procedure until we reach the end of $P$. This algorithm runs in $O(n)$ time and returns the minimal number $k$ of $x$ - or $y$-monotone subpaths, indicated by Proposition 1 .

Proposition 1. The greedy path splitting algorithm divides the input path $P$ into a minimal number of $x$ - or $y$-monotone subpaths in linear time.

Proof. Assume $\left(L_{1}, \ldots, L_{l}\right)$ is an optimal solution that divides $P$ into $l x$ - or $y$ monotone subpaths, and let $\left(P_{1}, \ldots, P_{k}\right)$ be the solution obtained by the above algorithm. Observe that due to the greedy approach the following two statements hold: (i) the path $L_{i}$ for the smallest $i$ such that $L_{i}$ differs from $P_{i}$ contains less vertices than $P_{i}$; (ii) there cannot be any path $L_{m}$ that fully contains some path $P_{j}=\left(v_{p}, \ldots, v_{q}\right)$ and also $v_{q+1} \in L_{m}$. This implies that if there is a path $L_{m}$ that contains $P_{j}$ they both share the same last vertex. Combined, we get that there is no sequence of paths $L_{1}, \ldots, L_{i}$ containing more vertices than the sequence $P_{1}, \ldots, P_{i}$ and hence $k \leq l$.

After splitting the input path, we schematize each subpath $\left(P_{i}, \pi_{i}\right)$ according to Section 3. We obtain a $\mathcal{C}$-oriented and orthogonal-order preserving embedding $\rho_{i}$ with minimum schematization cost and minimum path length for each $\left(P_{i}, \pi_{i}\right)$. For concatenating these subpaths, we must solve the following problem. 
Problem 2. Given a sequence of $k$ embedded $x$ - or $y$-monotone paths $\left(P_{i}, \rho_{i}\right)$ with $1 \leq i \leq k$, find an embedding $\xi$ of $P^{\prime}=P_{1} \oplus \cdots \oplus P_{k}$, where $\oplus$ denotes the concatenation of paths, such that

(i) for each subpath $\left(P_{i}, \xi_{i}\right)$, the embedding $\xi_{i}$ is a translation of $\rho_{i}$ and

(ii) $\left(P^{\prime}, \xi\right)$ is a simple $\mathcal{C}$-oriented path.

Our approach is based on iteratively embedding the subpaths $P_{1}, \ldots, P_{k}$. We ensure that in each iteration $i$ the embedding of $P_{1} \oplus \ldots \oplus P_{i}$ remains conflict-free, i. e., it has no self-intersections. We achieve this by adding up to three new pathlink edges between any two adjacent subpaths $P_{i}$ and $P_{i+1}$. For each $1 \leq i \leq k$ let $B_{i}$ denote the bounding box of $\left(P_{i}, \rho_{i}\right)$. We show how to construct an embedding $\xi$ of $P^{\prime}$ such that for any $i \neq j$ we have $B_{i} \cap B_{j}=\emptyset$. Consequently, since each individual $\left(P_{i}, \rho_{i}\right)$ is conflict-free, $\left(P^{\prime}, \xi\right)$ is conflict-free as well. A key operation of the algorithm is shifting a subpath $P_{i}$ (or equivalently a bounding box $B_{i}$ ) by an offset $\Delta=\left(\Delta_{x}, \Delta_{y}\right) \in \mathbb{R}^{2}$. This is done by defining the lower left corner of each bounding box $B_{i}$ as its origin $o_{i}$ and storing the coordinates of $P_{i}$ relative to $o_{i}$, i. e., $\xi(v)=o_{i}+\rho_{i}(v)$. Note that shifting preserves all local properties of $\left(P_{i}, \rho_{i}\right)$, i. e., the orthogonal order as well as edge lengths and orientations.

Each iteration of our algorithm consists of two steps. First, we attach the subpath $P_{i}$ to its predecessor $P_{i-1}$. To that end, we initially place $\left(P_{i}, \xi_{i}\right)$ such that the last vertex $u$ of $P_{i-1}$ and the first vertex $v$ of $P_{i}$ coincide. Then we add either two path-link edges (if the monotonicity directions of $P_{i-1}$ and $P_{i}$ are orthogonal) or three path-link edges (if $P_{i-1}$ runs in the opposite direction of $P_{i}$ ) between $u$ and $v$ and shift $B_{i}$ by finding appropriate lengths for the new edges such that $B_{i-1} \cap B_{i}=\emptyset$. Paths $P_{i-1}$ and $P_{i}$ are now conflict-free, but there may still exist conflicts between $P_{i}$ and paths $P_{j}(j<i-1)$. These are resolved in a second step that, roughly speaking, "inflates" $B_{i}$ starting at $v$ until it has reached its original size. Any conflicting bounding boxes are "pushed" away from $B_{i}$ by stretching some of the path-link edges. In the following, we explain our procedures for attaching a subpath and resolving conflicts in more detail.

Attaching a Subpath. Without loss of generality, we restrict ourselves to the case that $P_{i-1}$ is an $x$-monotone path from left to right. Let $u$ be the last vertex of $P_{i-1}$ and $v$ be the first vertex of $P_{i}$. If $P_{i}$ is $y$-monotone we add a horizontal edge $e_{1}=u u^{\prime}$ with $\alpha_{\xi}\left(e_{1}\right)=0^{\circ}$ connecting $u$ to a new vertex $u^{\prime}$. Then we also add a vertical edge $e_{2}=u^{\prime} v$ with $\alpha_{\xi}\left(e_{1}\right)=90^{\circ}$ if $P_{i}$ is upward directed and $\alpha_{\xi}\left(e_{1}\right)=270^{\circ}$ if it is a downward path. Otherwise, if $P_{i}$ is $x$-monotone from right to left, we add two vertices $u^{\prime}$ and $u^{\prime \prime}$ and three path-link edges $e_{1}=u u^{\prime}$, $e_{2}=u^{\prime} u^{\prime \prime}$, and $e_{3}=u^{\prime \prime} v$ with $\alpha_{\xi}\left(e_{1}\right)=0^{\circ}, \alpha_{\xi}\left(e_{2}\right)=90^{\circ}$ if $P_{i}$ is above $P_{i-1}$ in $\pi$ or $\alpha_{\xi}\left(e_{2}\right)=270^{\circ}$ otherwise, and $\alpha_{\xi}\left(e_{3}\right)=180^{\circ}$. Note that technically we treat each path-link edge as having its own bounding box with zero width or height. It remains to set the lengths of the path-link edges such that $B_{i} \cap B_{j}=\emptyset$ by computing the vertical and horizontal overlap of $B_{i-1}$ and $B_{i}$. Figure 3 illustrates both situations. 


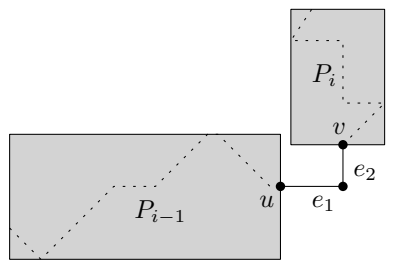

(a) Paths with orthogonal directions

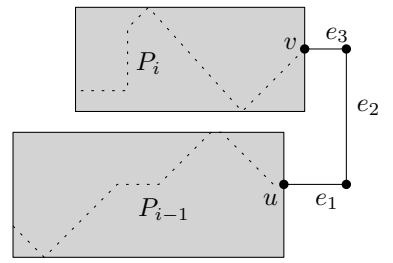

(b) Oppositely directed paths

Fig. 3: Two examples for attaching $P_{i}$ to $P_{i-1}$ by inserting path-link edges.

Resolving Conflicts. After adding $P_{i}$ we have $B_{i-1} \cap B_{i}=\emptyset$. However, there may still exist conflicts with any $B_{j}, 1 \leq j<i-1$. In order to free up the space required to actually place $B_{i}$ without overlapping any other bounding box, we push away all conflicting boxes in three steps. For illustration, let $P_{i}$ be $x$-monotone from left to right, and let $v$ be the first vertex of $P_{i}$.

Each bounding box $B$ is defined by its lower left corner $l l(B)=\left(l l_{x}(B), l l_{y}(B)\right)$ and its upper right corner $u r(B)=\left(u r_{x}(B), u r_{y}(B)\right)$. In the first step we identify the leftmost box $B^{\prime}$ (if any) that is intersected by a line segment that extends from $\xi(v)$ to the right with length equal to the width of $B_{i}$. For this box $B^{\prime}$ we have $l l_{y}\left(B^{\prime}\right) \leq y_{\xi}(v) \leq u r_{y}\left(B^{\prime}\right)$ and $l l_{x}\left(B_{i}\right) \leq l l_{x}\left(B^{\prime}\right) \leq u r_{x}\left(B_{i}\right)$. If there is such a $B^{\prime}$ let the offset be $\Delta_{x}=u r_{x}\left(B_{i}\right)-l l_{x}\left(B^{\prime}\right)$.

Now we shift all bounding boxes $B$ that lie completely to the right of $l l_{x}\left(B^{\prime}\right)$ to the right by $\Delta_{x}$. All horizontal path-link edges (which are also considered bounding boxes by themselves) that connect a shifted with a non-shifted path are stretched by $\Delta_{x}$ to keep the two paths connected. Note that there is always a horizontal path-link edge between any two subsequent paths.

Next, we inflate $B_{i}$, which is currently a horizontal line segment, downwards: we first determine the topmost conflicting box $B^{\prime \prime}$ (if any) below a horizontal line through $\xi(v)$, i. e., a box $B^{\prime \prime}$ whose $x$-range intersects the $x$-range of $B_{i}$ and for which $l l_{y}\left(B_{i}\right) \leq u r_{y}\left(B^{\prime \prime}\right) \leq y_{\xi}(v)$. If we find such a $B^{\prime \prime}$ we define the vertical offset $\Delta_{y 1}=u r_{y}\left(B^{\prime \prime}\right)-l l_{y}\left(B_{i}\right)$. We shift all bounding boxes $B$ that lie completely below $u r_{y}\left(B^{\prime \prime}\right)$ downwards by $\Delta_{y 1}$. All vertical path-link edges that connect a shifted with a non-shifted box are stretched by $\Delta_{y 1}$ in order to keep the two boxes connected. Again, there is always a vertical path-link edge between any two subsequent paths. Finally, we inflate $B_{i}$ upwards, which is analogous to the downward inflation. Figure 4 shows an example.

The approach explained above first inflates the box to the right, then downwards, and finally upwards. Of course, we could use any strategy of inflating the box. In fact, since different strategies yield different results, we could test a fixed number of strategies and keep the result that minimizes the overall increase in edge lengths.

In the following, we show that our algorithm indeed solves Problem 2. 
Lemma 2. Our algorithm computes a conflict-free embedding $\xi$ of $P^{\prime}=P_{1} \oplus$ $\cdots \oplus P_{k}$.

Proof. We prove the theorem by induction. By definition, fhe first path $P_{1}$ has a conflict-free embedding. Now, assume that $P_{1} \oplus \cdots \oplus P_{i-1}$ is already embedded conflict-free. We attach $P_{i}$ to $P_{i-1}$, such that $B_{i} \cap B_{i-1}=\emptyset$ holds. Thus, $P_{i}$ and $P_{i-1}$ do not have a conflict. Next, we shift all $B_{j}$ with $j<i$ such that $B_{i} \cap B_{j}=\emptyset$ in the end.

What remains to be shown is that our shifting-operation does not create new conflicts between any two boxes $B_{j}$ and $B_{j^{\prime}}$ with $j, j^{\prime}<i$. Consider, e. g., a shift to the right and assume that after the shift $B_{j}$ and $B_{j^{\prime}}$ intersect, while they did not intersect before the shift. Clearly, if none of the boxes has been moved, they cannot intersect. So either the shift moved both boxes by the same offset $\Delta_{x}$, or it moved only the rightmost of the two boxes to the right by $\Delta_{x}$. There is no vertical movement. So in both cases the horizontal distance of the boxes does not decrease and it is impossible that $B_{j}$ and $B_{j^{\prime}}$ intersect after the shift. The same observation holds for the vertical shifts.

Theorem 4. Our algorithm computes a solution $\left(P^{\prime}, \xi\right)$ to Problem 2 by adding at most $3(k-1)$ path-link edges to $P$. It can be implemented with a running time of $O\left(k^{2}+n\right)$.

Proof. The correctness of the algorithm follows from Lemma 2 and due to the fact that the embeddings $\left(P_{i}, \rho_{i}\right)$ within the bounding boxes $B_{i}$ remain unchanged. Clearly, we add at most three edges between any two subsequent paths, which shows the bound on the number of path-link edges.

It remains to show the running time of $O\left(k^{2}+n\right)$. For the position of each bounding box we maintain the coordinates of its lower left and upper right corners. In each iteration, attaching a new subpath, requires constant time since it

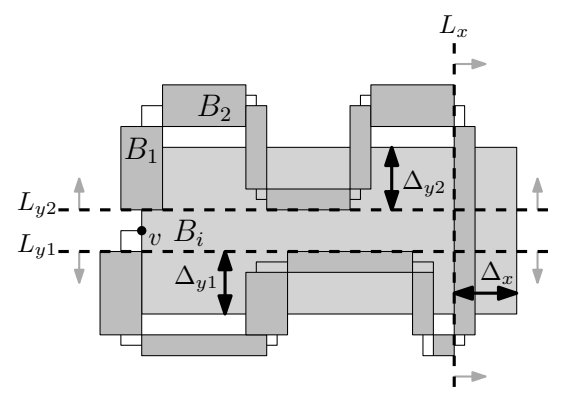

(a) Before resolving the conflict

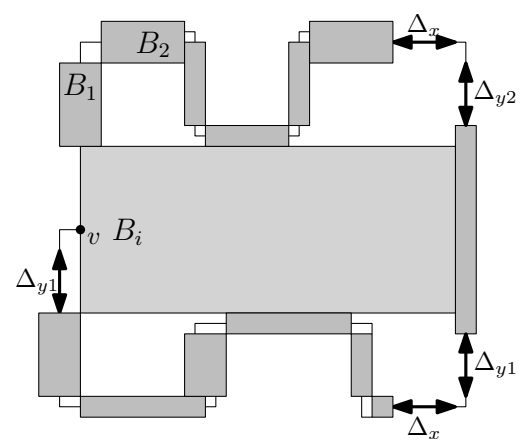

(b) After resolving the conflict

Fig. 4: Example for iteratively resolving conflicts induced by attaching $P_{i}$. First, we shift everything right of $L_{x}$ to the right by $\Delta_{x}$. Then, we shift everything below $L_{y 1}$ by $\Delta_{y 1}$ downward, and finally, we shift everything above $L_{y 2}$ upward. 
depends only on the overlap of the new bounding box and its immediate predecessor. Resolving all conflicts requires $O(k)$ time per iteration: When adding path $P_{i}$ we need to check for each box $B_{j}(j<i-1)$ and the boxes of their respective path-link edges if and how they conflict with $B_{i}$. Once the required offset is computed, we shift $O(k)$ boxes by updating their lower left and upper right corners, as well as their origins. This is done in constant time per box and $O(k)$ time in total per iteration. So for all $k$ iterations the running time is $O\left(k^{2}\right)$. Finally, we obtain the embedding $\xi$ of $P^{\prime}$ by computing the absolute coordinates of all vertices in $O(n)$ time.

Note that we can improve the running time if the number $I$ of occurring conflicts and shifts is small. More precisely, if $I<k^{2} / \log ^{2} k$ we can use dynamic interval trees and dynamic binary search trees to store the segments and coordinates of all bounding boxes [3]. In this case, obtaining the bounding boxes that define the offsets for all shift operations can be done in $O\left(k \log ^{2} k+I\right)$ total time. Each stretch or shift of a box requires an update in these data structures, which can be done in $O\left(\log ^{2} k\right)$ time. Hence, for $I$ updates and $k$ queries we obtain a total running time of $O\left((I+k) \log ^{2} k+n\right)$ instead.

\section{Conclusion}

We presented a novel two-step approach to schematize monotone paths motivated from drawing route sketches. We embed the path in a $\mathcal{C}$-oriented way such that the orthogonal ordering is preserved followed by optimizing the edge lengths. Our approach has polynomial running time and is dominated by the optimization of the edge lengths using linear programming. In order to use our approach for general simple paths we proposed a three-step heuristic that first decomposes the path (of length $n$ ) into $k$ monotone subpaths, embeds all of them independently, and finally reconcatenates them in such a way that the resulting embedding is $\mathcal{C}$-oriented and crossing-free. Preliminary results from an implementation of our approach (see Fig. 1c) indicate that typically $n \leq 100$ for the road network of Germany, making our approach very efficient for drawing route sketches in practice. Moreover, our experiments also indicate that $k$ tends to be no larger than 3 .

However, for usage in a real-world application, some problems remain. We need to label the edges, indicate turns, etc.; we plan to tackle all these problems. Moreover, it would be interesting to optimize the routes for simplicity in terms of driving and visualization rather than solely for travel time, i. e., a slightly longer route with less road changes might be preferable as it lowers the chance of making mistakes. The running time of our approach is currently dominated by the time required for solving the LP that optimizes the edge lengths. It would be interesting to find an algorithm solving this problem faster. Apart from schematizing a single route, it is another interesting problem to draw a whole set of alternative routes in a single sketch. 


\section{Acknowledgments}

We thank David Eppstein, Bastian Katz, Maarten Löffler, Ignaz Rutter, and Markus Völker for discussions on the edge-length minimization problem.

\section{References}

1. M. Agrawala and C. Stolte. Rendering effective route maps: Improving usability through generalization. In E. Fiume, editor, Proc. 28th Ann. Conf. Computer Graphics and Interactive Techniques (SIGGRAPH'01), pages 241-249. ACM, 2001.

2. U. Brandes and B. Pampel. On the hardness of orthogonal-order preserving graph drawing. In I. G. Tollis and M. Patrignani, editors, Proc. 16th Internat. Symp. Graph Drawing (GD'08), volume 5417 of Lecture Notes Comput. Sci., pages 266277. Springer-Verlag, 2009.

3. M. de Berg, O. Cheong, M. van Kreveld, and M. Overmars. Computational Geometry: Algorithms and Applications. Springer-Verlag, third edition, 2008.

4. T. Dwyer, Y. Koren, and K. Marriott. Stress majorization with orthogonal ordering constraints. In P. Healy and N. S. Nikolov, editors, Proc. 13th Internat. Symp. Graph Drawing (GD'05), volume 3843 of Lecture Notes Comput. Sci., pages 141152. Springer-Verlag, 2006.

5. D. Merrick and J. Gudmundsson. Path simplification for metro map layout. In M. Kaufmann and D. Wagner, editors, Proc. 14th Internat. Symp. Graph Drawing (GD'06), volume 4372 of Lecture Notes Comput. Sci., pages 258-269. SpringerVerlag, 2007.

6. K. Misue, P. Eades, W. Lai, and K. Sugiyama. Layout adjustment and the mental map. J. Visual Languages and Computing, 6(2):183-210, 1995.

7. G. Neyer. Line simplification with restricted orientations. In F. K. Dehne, A. Gupta, J.-R. Sack, and R. Tamassia, editors, Proc. 6th Int. Workshop on Algorithms and Data Structures (WADS'99), volume 1663 of Lecture Notes Comput. Sci., pages 13-24. Springer-Verlag, 1999.

8. M. Ovenden. Metro Maps of the World. Capital Transport Publishing, 2003.

9. B. Tversky. Cognitive maps, cognitive collages, and spatial mental models. In Proc. 1st Conference on Spatial Information Theory (COSIT'93), volume 716 of Lecture Notes Comput. Sci., pages 14-24. Springer-Verlag, 1993.

10. B. Tversky and P. U. Lee. Pictorial and verbal tools for conveying routes. In Proc. 4th Conference on Spatial Information Theory (COSIT'99), volume 1661 of Lecture Notes Comput. Sci., pages 51-64. Springer-Verlag, 1999.

11. P. Vaidya. Speeding-up linear programming using fast matrix multiplication. In Proc. 30th Annual Symposium on Foundations of Computer Science (FOCS'89), pages 332-337, 1989. 